\title{
CONTRIBUCIÓN DE LA TECNOLOGÍA SENSORES DE MOVIMIENTO EN LA PRODUCCIÓN DE VIDEOS EDUCATIVOS PARA LA EDUCACIÓN BÁSICA. DISEÑO DE UN SOFTWARE DE CONTROL GESTUAL
}

\author{
CONTRIBUTION OF MOTION SENSING TECHNOLOGY IN THE PRODUCTION OF \\ EDUCATIONAL VIDEOS FOR BASIC EDUCATION. \\ DESIGN OF A GESTURAL CONTROL SOFTWARE
}

\section{WELLINGTON VILLOTA OYAVIDE', YAMIL LAMBERT SARANGO²}

1 Universidad Católica de Santiago de Guayaquil, Ecuador. wellington.villota@cu.ucsg.edu.ec

2 Universidad Católica de Santiago de Guayaquil, Ecuador. yamil.lambert@cu.ucsg.edu.ec

La tecnología sensores de movimientos, está siendo cada vez más utilizada en interfaces de usuario como elemento de interacción entre el usuario y el software. El objetivo del estudio es analizar la contribución de la tecnología sensores de movimientos en la producción de multimedios interactivos educativos, realizando un experimento de corte técnico-pedagógico a partir de los drivers controladores, el kit de desarrollo Software Development Kity el software de autoría, para diseñar un software de Control Gestual. Se aplicó el método experimental para conectar el sensor Kinect al puerto USB de la computadora para efectos de probar la detección del movimiento de una persona y determinar que se ha instalado correctamente. Se seleccionó a diez usuarios con experiencia multimedia y diez niños de sexto de Educación Básica, para determinar el grado de usabilidad de la aplicación desarrollada mediante observaciones y encuestas. La tasa de respuesta indicó que el $32 \%$ prefiere usar videos o videojuegos en el aprendizaje, y el $27 \%$ logra la interactividad. Se concluye que la creación de aplicaciones multimedia debe basarse en la usabilidad de videos como contenido novedoso, y en la interactividad, mediante sensores de movimiento como elemento de fácil interacción entre el usuario y el software.

PALABRAS CLAVE: control gestual, multimedia, sensor de movimientos, usabilidad, videos educativos .

KEYWORDS: gestural control, multimedia, motion sensor, usability, educational videos. an element of interaction between the user and the software. The aim of the study is to analyze the contribution of sensor technology movements in the production of educational interactive multimedia, performing an experiment of technical and pedagogical cut from controllers drivers, the development kit SDK and authoring software to design Gestural Control software. The experimental method was applied to connect the Kinect sensor to the USB port of the computer to test the effects of motion detection of a person and determine that is installed correctly. Ten users were selected multimedia experience and ten children 6 th of Basic Education were selected to determine the degree of usability of the application developed by observations and surveys. The response rate was $32 \%$ prefer to use video or video games in learning and the "Interactivity" with 27\%. It is concluded that the creation of multimedia applications should be based on the usability of videos like new content and interactivity by motion sensors as part of easy interaction between the user and the software. 
INTRODUCCIÓN

A través del desarrollo de la multimedia se ha dado mucha atención a la creación de aplicaciones interactivas por el contenido dinámico que poseen a través del texto, audio, video e imágenes, al igual que su usabilidad; sin embargo, estas aplicaciones aun siendo novedosas para los usuarios, tienen mayor dificultad en su operación, principalmente aquellas que utilizan controles tradicionales como dispositivo de entrada de datos, mediante mouse, teclado y pantallas touch.

Figueroa (2012) con el objetivo de encontrar la interacción humana natural ideal como campo de estudio Interacción Hombre-Máquina (HCI) y además, por medio de gestos, donde plantean las ventajas de un usuario que interactúa con un sistema usando movimientos naturales, lo que implica ciertos problemas no resueltos como el desempeño en tiempo y la tasa de reconocimiento.

Rodríguez (2013) realizó una investigación sobre los requerimientos, características y necesidades de una solución, así como las posibilidades y herramientas que ofrece un sistema computacional cada vez más avanzado, amplio y completo.

Con el avance tecnológico, las posibilidades de interacción con los sistemas computacionales modernos han venido ampliándose y tomando rutas y formas que hace apenas unos años no se consideraban posibles. Rodríguez (2013) autorreseña en su trabajo los esquemas iniciales de entrada-salida de datos, pasando por el uso de tarjetas perforadas o interruptores y alertas luminosas o sistemas de impresión, hasta el desarrollo del teclado y el mouse, y las interacciones que utilizan esquemas multitoque e interacciones naturales.

La interacción humano-computador es un campo de crecimiento constante, pero plantea un vacío en términos de esquemas de interacción para escenarios de creación artística y de enseñanza, el autor propone la utilización de Kinect como dispositivo de interacción humano-máquina para los escenarios descritos, teniendo en cuenta su accesibilidad, robustez, facilidad de uso y modificación, así como la amplísima gama de posibilidades que ofrece.

Chuya et al.(2013) analizó el Sistema de Movimiento Humano usando sensores Kinect (SAM), integrado por tres bloques principales: captura de movimiento, reconstrucción, modelado y animación 3D. Su objetivo se basa en la captura de las trayectorias, análisis estadísticos, visua- lización gráfica de planos cinemáticos del desplazamiento y la animación 3D en tiempo real.

González de Dios (2014) presentó un sistema de reconocimiento de gestos para teleoperar robots, basado en el sensor Kinect. El autor dividió en dos partes su investigación: la primera, relativa al diseño y evaluación de un sistema de reconocimiento de gestos basado en el sensor Kinect; y la segunda, relativa a la tele operación de robots usando el sistema de reconocimiento de gestos desarrollado.

El objetivo del autor en la primera parte, es enumerar las características y limitaciones del sensor Kinect. Posteriormente, analiza la detección de movimiento y se presenta la máquina de estados propuesta para detectar el movimiento de un gesto. Explica los posibles escaneos de un esqueleto en 3 dimensiones para mejorar la detección de gestos y el algoritmo utilizado para la detección de gestos; además del algoritmo de alineamiento temporal dinámico (DTW).

En la segunda parte, el autor expone el sistema de teleoperación del robot y su integración con el evaluador de gestos: este sistema controla el robot Lego Mindstorm mediante la detección de gestos o el reconocimiento de voz (González de Dios, 2014).

La aparición de las nuevas tecnologías revolucionaron la forma en que interactuábamos con las máquinas, un gran ejemplo de ello son los equipos con pantalla táctil que actualmente son muy comunes. Ahora tenemos el control gestual que se encuentra en expansión (Cortes, Sandoval y Trujillo, 2014).

Debido a que los sistemas multimedia actuales usan los tradicionales dispositivos de control en sus sistemas de menú como el mouse y el teclado, considerados en estos días totalmente obsoletos, con un $35 \%$ de uso respectivamente, y la experiencia de usuario a nivel usabilidad es deficiente, ellos se inclinan a ver y experimentar nuevas formas de interactuar, en las que los sensores de movimiento se convierten en protagonistas principales por su fácil adaptación y manejo, y donde el ser humano es el nuevo elemento de interacción con el software mediante sus movimientos y gestos.

El sensor Kinect, creado con el único propósito de revolucionar la experiencia del usuario al momento de controlar la consola de videojuegos Xbox 360 mediante gestos corporales o comandos de voz, ha diversificado su uso pasando de videojuegos a sistemas informáticos y aplicaciones multimedia. 
Por lo tanto, el propósito de este estudio es analizar la contribución de la tecnología sensores de movimiento en la producción de multimedios interactivos educativos, para determinar si mediante el uso del sensor Kinect como elemento de interacción entre el ser humano y la aplicación, mejora la usabilidad de operación del software multimedia y la experiencia de usuario.

\section{METODOLOGÍA}

El tipo de investigación seleccionada es el descriptivo. Se utilizó un enfoque mixto, cuantitativo y cualitativo con un método experimental. Para la recolección de datos se usó análisis documental bibliográfico, encuestas y observaciones. El objetivo general fue analizar la contribución de la tecnología sensores de movimiento en la producción de multimedios interactivos educativos; en este sentido, se buscó identificar y caracterizar cómo puede ayudar esta tecnología en la producción de multimedios interactivos educativos, resaltando las cualidades y características de ésta.

La población en estudio estuvo conformada por 50 usuarios con experiencia multimedia, con una edad comprendida entre los 25 y 50 años, además de veinticinco niños de sexto grado de Educación Básica media de la Unidad Educativa Santiago Mayor de Guayaquil, para un total 75 personas entre expertos y novatos en aplicaciones multimedia. Se seleccionó a 10 usuarios con experiencia multimedia y 10 niños para aplicar un muestreo intencional, a través del método experimental, se excluyeron a usuarios docentes en estado adulto tardío o adulto mayor y tercera edad, que carecen de habilidades y destrezas en el manejo de tecnologías de información.

\section{Se utilizaron:}

MATERIALES

- Un computador personal para recibir la señal (la información captada) por el sensor Kinect y se la utiliza para el desarrollo y pruebas. También se encarga de tratar esa información. Un sensor de movimiento Kinect para Xbox 360 como herramienta principal del proyecto, que posee un servomotor, una cámara RGB, una cámara de profundidad y cuatro micrófonos (Fernández, 2012). Las especificaciones técnicas de hardware de Kinect se detallan en la tabla 1.
TABLA 1. ESPECIFICACIONES TÉCNICAS DEL KINECT

\begin{tabular}{|c|c|}
\hline COMPONENTES & CARACTERÍSTICAS \\
\hline Sensores & $\begin{array}{l}\text { - Lentes con sensores de color y profundidad } \\
\text { - Array de micrófonos para voz } \\
\text { - Tilt motorizado para ajuste del sensor }\end{array}$ \\
\hline Campo de visión & $\begin{array}{l}\text { - Campo de visión horizontal: } 57 \text { grados } \\
\text { - Campo de visión vertical: } 43 \text { grados } \\
\text { - Rango de tilt físico: } \pm 27 \text { grados } \\
\text { - Rango del sensor de profundidad: } 1.2 \mathrm{~m}-3.5 \mathrm{~m}\end{array}$ \\
\hline Despliegue de datos & $\begin{array}{l}\text { - } 320 \times 240 \text { profundidad de } 16 \text {-bit a } 30 \text { FPS } \\
\text { - } 640 \times 480 \text { color de } 32 \text {-bit a } 30 \text { FPS } \\
\text { - } 16 \text {-bit audio @ } 16 \mathrm{khz}\end{array}$ \\
\hline
\end{tabular}

Nota: Kinect for Windows SDK Programming Guide, 2012

- Un Vehículo Aéreo no tripulado (VANT), utilizado para producir cortos educativos con tomas áreas de los lugares turísticos de Guayaquil, es una aeronave que vuela sin tripulación que tiene una variedad de formas, tamaños, configuraciones y características (Zubair, 2009).

- Un sensor Microsoft Kinect para Windows SDK que nos permitió utilizar las librerías del sensor para la recolección de datos y control de eventos. Su sintaxis se deriva de otro lenguaje de programación llamado $\mathrm{C}+\mathrm{C}++$ pero con muchas mejoras, tales como: seguridad, recolección de datos entre otros (Hejlsberg, Wiltamuth, y Golde, 2006).

- Microsoft Visual Studio Express cómo un entorno de desarrollo para crear el software de Control Gestual. Permite la edición para crear y entregar experiencias de inmersión web, aplicaciones, juegos y contenidos multimedia (Gousset et al., 2014).

- Guión multimedia con una estructura altamente útil para desarrollar una aplicación de forma óptica ya que permite estimar recursos técnicos y humanos (Bou, 1997).

- Storyboard con una serie de gráficos en secuencia que muestra de forma ilustrada cómo se imagina el director de un video la forma en que quedará la producción final (Sáenz, 2008).

- Adobe Premiere como software de edición no lineal de alto rendimiento para los profesionales del vídeo (Adobe Creative Team, 2012).

Se aplicó una encuesta de 10 ítems con alternativas de respuesta con escala de likert: total en desacuerdo, en desacuerdo, neutral, de acuerdo y totalmente de acuerdo. El mismo fue validado a través del juicio de 10 expertos docentes en 
multimedia de la Universidad Católica de Santiago de Guayaquil, y diez 10 niños de sexto grado de educación básica media de la Unidad Educativa Santiago Mayor de Guayaquil.

El análisis de la información se realizó a través de estadística descriptiva y tabulando con Microsoft Excel. Los datos recogidos recibieron un tratamiento descriptivo, para conocer datos de los docentes y estudiantes según las categorías seleccionadas.

La decisión de tomar la encuesta desde esta perspectiva responde a que se obtienen suficientes características del contexto y sus participantes en poco tiempo, útil para esta circunstancia de inicio y final. Los datos categóricos que se obtuvieron son representables a través de tablas de frecuencia y tablas de contingencia, suficientes para realizar el análisis respectivo, y tener una visión bastante amplia del caso.

MÉTODOS

Con un método experimental se conectó el sensor Kinect al puerto USB de la computadora para efectos de probar la detección del movimiento de una persona y determinar que se ha instalado correctamente. Se documentaron los pasos necesarios para instalar y operar el sensor Kinect en un algoritmo, como se muestra en la figura 1 y 2. Luego se experimentó con el dispositivo para considerar su cámara RGB, el sensor de profundidad, el micrófono de múltiples matrices y su procesador personalizado que ejecuta el software patentado por Microsoft, que proporciona captura de movimiento de todo el cuerpo en 3D.

INICIO PROGRAMA

Instalar Microsoft Visual Studio 2013 Express o cualquier versión de Visual Studio 2013

Instalar Windows Software Development Kit for Windows 7

Instalar Mocrosoft_NET Framework 4.0

Si Windows no está actualizado Entonces

FINSI

SI está conectado el dispositivo kinict al puerto USB al computador ENTONCES Desconectar el dispositovo Kinect del puerto USB

FINSI SISDK de Kinect no está descargado Entonces

Ir a la página de descarga del SDK de Kinect para Windwos, hacer clic en la opción de Download

Guardar en el computador para instalarlo más adelante, clic en Guardar

FINS

Conectar la fuente de alimentación del sensor Kinect a la red eléctrica

Conectar Kinect en un puerto USB libre del PC y espere a que se reconozca los componentes del senso

SI Kinect está bien instalado ENTONCES

Debe ver parpadear el LED verde en el sensor de Kinect

En el administrador de dispositivo del panel de control, debajo de Michosoft Kinect deben aparecer los siguientes modos:

Microsoft Kinect audio Array Control

Microsoft Kinect Camara

FINSI

Microsoft Kinect Device

FIN PROGRAM

Figura 1. Algoritmo Seudocódigo de instalación del sensor Microsoft Kinect.

INICIO PROGRAM

Abriremos Visual Studio 2013

Crear un proyecto WPF (Windows Presentación Foundation ) y le damos un nombre y aceptamos

En el explorador de proyectos desplegar el modo de Main window.xaml y acceder al fichero MainWindow-xams.cs

Presionamos con el botón derecho del ratón encima de "References " elegimos "añadir referencia"

Sobre el cuadro de diálogo que se muestra seleccionaremos la referencia al SDK "Microsost.Research.Kinect" y aceptamos

Con la referencia agragada añadiremos el espacio de nombres a junto con los demás usings de nuestro fichero para poder utilizar las

claves y métodos de SDK

Dentro del constructor (método MainWindow()) de nuestra ventana añadiremos el evento Loaded, donde pondremos el código para

inicializar

el sensor, y el evento Closed para finalizar el uso

Crearemos un atributo de la clase Runtime, clase que hace referencia al sensor Kinect y que instanciaremos e inicializaremos más

adelante

En el método MainWindow_Loaded creamos la instancia para controlar el sensor e inicializaremos el sensor con las opciones que

queremos usar que pueden ser:

Cámara RCB

Profundidad Skeletal

Tracking

En el método MainWindow_Closed finalizaremos el uso del sensor. Cuando se cierra la aplicación y no lo estaremos usando

FIN PROGRAMA

Figura 2. Algoritmo seudocódigo de operación del sensor Microsoft Kinect. 
Se analizó el reconocimiento facial y de voz haciendo una prueba con el micrófono de matrices del sensor de Kinect, que permite a la computadora llevar a cabo la localización de la fuente acústica y la supresión del ruido ambiente y su aporte a la aplicación.

A través de una encuesta a expertos en el área multimedia, seleccionados de manera intencional, se conoció la opinión de la experiencia de usuario de cada uno de ellos sobre el producto multimedia interactivo que es controlado a través de gestos. Además, se hizo la validación de la encuesta; para ello se desarrollaron actividades con grupos de docentes y con expertos de la academia de la Universidad Católica de Santiago de Guayaquil. Estas actividades correspondieron a jornadas de trabajo y reuniones en las cuales se les dio a conocer el desarrollo de la encuesta, sus objetivos, las preguntas que buscamos responder, sus dimensiones y contenidos.

Se escribió un guión multimedia con una descripción detallada de todas y cada una de las escenas del producto interactivo. Se estructuraron todas las pantallas de la aplicación informática multimedia.

También se creó un storyboard con cada una de las pantallas, su descripción de los elementos sensibles a la interacción del usuario y su comportamiento. Además se explica el comportamiento de los elementos que no dependen del usuario para activarse, por ejemplo: animaciones, sonidos que se cargan con la aplicación.

Se escribieron guiones audiovisuales para la producción de los videos con una descripción detallada de todas y cada una de las partes del corto educativo que permita verificar la integración sensor-video. El guión incluye el sonido ambiental suave, la voz del narrador, el tiempo en minutos y la toma aérea.

$\mathrm{El}$ producto multimedia es operado por medio de gestos para reproducir, detener y cambiar de video desde la galería a través de un sistema de menú, hasta determinar los parámetros óptimos de funcionamiento.

El producto no utiliza sistemas de mando o dispositivos de entrada de las GUI como sería un ratón, teclado alfanumérico, lápiz óptico, touchpad, joystick etc. y en su lugar, se hace uso de movimientos gestuales, tales como las manos o el cuerpo es el mismo mando de control. Se aplicó el reconocimiento de gestos a la aplicación multimedia en la que se utilizó técnicas de procesamiento y análisis de series temporales para hacer que el computador entienda un gesto.

RESULTADOS

En el primer experimento se analiza la información de operacionalizar el uso de la tecnología sensores de movimientos, conectando los sensores a una computadora, para elaborar un algoritmo - seudocódigo de instalación y operación. Los resultados obtenidos en el primer momento de la investigación siguen el mismo orden en que fueron experimentados y cómo se presentan en el diseño de la metodología, por lo que se elaboró un algoritmo - seudocódigo de instalación y operación

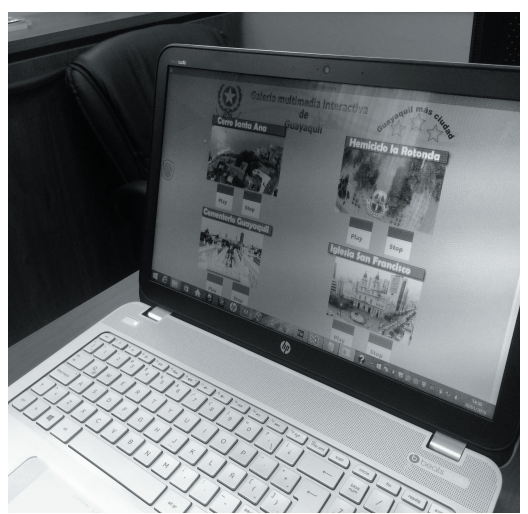

Figura 4. Pruebas del producto multimedia interactivo haciendo puño con la mano para activar el scroll de la aplicación. 
del sensor Microsoft Kinect, lo que queda evidenciado en un producto concreto como es el documento que contiene la metodología de trabajo con los pasos del algoritmo - seudocódigo de instalación y operación del sensor Microsoft Kinect.

En el segundo experimento se analiza la información producto de la experimentación para proceder a establecer los parámetros técnicos requeridos para la producción de multimedios interactivos educativos controlados con sensores, realizando una investigación bibliográfica y consulta a expertos, para formular una metodología de trabajo.

En el tercer experimento de la investigación se realizan o producen 10 cortos educativos, a partir de guiones originales escritos por miembros del equipo investigador, usando filmaciones normales y tomas áreas de drones, que permitan la realización del experimento de acople sensor-video, el resultado de esta etapa son cortos educativos que incluyen tomas áreas. El producto concreto que se obtuvo en esta etapa del proyecto es el documento con los guiones y el storyboard para 10 cortos educativos con una duración de 5 a 10 minutos.

En el cuarto experimento se realiza una prueba de acople de un producto multimedia usando sensores de movimiento para controlar la galería de videos previamente producidos, para diseñar un Software de Control Gestual. Los resultados obtenidos en esta etapa de la investigación siguen el mismo orden en que fueron $\mathrm{ex}^{-}$ perimentados y como se presentan en el diseño de la metodología se evidencia en la realización practica de un experimento de acople entre el producto multimedia y el sensor de movimiento Kinect. El resultado como producto concreto es un informe que incluye el guión multimedia principal y fuentes de programación del producto educativo entregable, que muestra una galería de videos que es controlado a través de los gestos de una persona o usuario.

Para la etapa final, se validó el impacto del Software de Control Gestual en la experiencia de usuarios, realizando demostraciones con un panel y una encuesta final, para determinar el porcentaje de usabilidad del producto multimedia y su impacto; esto permitió determinar que el sistema multimedia debería tener mínimo un $32 \%$ de porcentaje en la usabilidad.

Se desarrolló un sistema multimedia que posee un menú principal que sirve para que el usuario elija alguna de las principales funciones del programa. En este caso las opciones son los diferentes videos con las tomas de sitios importantes de la ciudad de Guayaquil. Puede ser controlado por teclado y mouse de forma tradicional, pero la característica principal es que funciona a través de sensores de movimiento Ms Kinect, un sistema que funciona con éxito en la industria del videojuego. La cámara podrá detectar nuestro cuerpo a una distancia mínima de 1.5 $\mathrm{m}$, lo que nos garantiza el vivir una experiencia totalmente diferente a la que estamos acostumbrados, con interfaces naturales e intuitivas.

Para la variable independiente "Tecnología sensores de movimiento", se utilizó una metodología experimental donde se prueban las cámaras, campo de visión, flujo de datos, sistema de seguimiento, sistema de audio del sensor; los resultados fueron medidos por cada dimensión, tal es el caso de la cámara para obtener imágenes en color y 2 cámaras de infrarrojos, el campo de visión con la detección de objetos es de $43^{\circ}$ verticales por $57^{\circ}$ horizontales, además el flujo de datos con la resolución de imágenes 640x480 a 32 bits de color es de 30 cuadros por segundo.

Para la variable dependiente "Producción de multimedios interactivos", se aplicaron algunas metodologías, tales como encuestas, creación de storyboard de forma gráfica y panel de expertos; se empleó como indicador el "mensaje comunicacional del producto multimedia" (conocer al cliente), la elaboración del patrón o secuencia detallada de todas y cada una de las escenas de la aplicación y su creación en función de los resultados de las pruebas del prototipo planteado como dimensión.

Para iniciar el diagnóstico se aplicó una encuesta a expertos en el área multimedia. Estos fueron seleccionados de manera intencional para conocer su la opinión sobre su "experiencia de usuario" del producto multimedia interactivo que será controlado a través de gestos. La encuesta que tiene por objetivo saber si los docentes manejan la tecnología de sensores y el impacto de la usabilidad en el aprendizaje de los estudiantes, si están satisfechos de enseñar sus materias con esta tecnología e iniciar la exploración de las necesidades normativas para el análisis.

El resultado final es el diseño y programación de un Software de Control Gestual a partir de la tecnología sensores de movimiento de Microsoft Kinect.

En la última etapa demostrativa y en coordinación con una docente de sexto de básica de la institución Colegio "Santiago Mayor" de la ciudad de Guayaquil se experimentó con 
estudiantes el prototipo de software multimedia conectado al computador por medio del sensor de movimiento, permitiéndoles controlar e interactuar con un sistema de menú con 10 videos de locaciones de la ciudad de Guayaquil, usando tomas áreas con drones.

La encuesta a los estudiantes y la información recogida con la aplicación de este instrumento, fue procesada con Microsoft Excel como software y aporta a la investigación la visión de entrada. Los datos recogidos reciben un tratamiento descriptivo debido al uso de la información en este momento como puerta de entrada al contexto para conocer datos de los docentes y estudiantes según las categorías seleccionadas. La decisión de tomar la encuesta desde esta perspectiva responde a que se obtienen suficientes características del contexto y sus participantes en poco tiempo, útil para esta circunstancia de inicio y final. Los datos categóricos que se obtuvieron son representables a través de tablas de frecuencia y tablas de contingencia, suficientes para realizar el análisis respectivo, y tener una visión bastante amplia del caso.

\section{DISCUSIÓN DE RESULTADOS}

La tecnología, como una herramienta a disposición de los maestros o docentes, al ser usada en el aula de clase, evidencia en los estudiantes no solo el interés por aprender, sino que produce un aprendizaje significativo en los conocimientos impartidos, y son los videos o videojuegos los de mayor preferencia para ellos con un $32 \%$ en primera posición; en segundo lugar se tiene la "Interactividad", con el 27\%; tanto la usabilidad como la interactividad son los de mayor importancia a la hora de crear aplicaciones multimedia.

A la hora de desarrollar aplicaciones multimedia interactivas que sean novedosas y atraigan la atención de los usuarios, se deben destacar dos factores importantes: 1) La usabilidad que debe tener el software creado para su fácil manejo y la comprensión que mediante el uso de videos produce una mayor motivación e interés por aprender por parte de los estudiantes. Es un error pensar que aun contando con un producto digital que haya sido creado con la última tecnología de software, este solucionará un problema o se constituirá como un aporte en el proceso de enseñanza-aprendizaje, si fueron olvidados la claridad y la elegancia que todo programa debe tener, produciendo efectividad, eficiencia y satisfacción en el contexto de uso. 2) La interactividad en los sistemas multimedia en un alto grado refleja la relación de participación activa entre los usuarios y los programas, donde su diseño planifica la navegación entre pantallas en las que el usuario sienta que realmente controla y maneja una aplicación. En este sentido el usuario navega por la aplicación y se siente libre.

Por lo expuesto hasta aquí, tanto la usabilidad como la interactividad son elementos a considerar a la hora de diseñar un software. Los sistemas multimedia que usan los tradicionales dispositivos de control en sus sistemas de menú, como el mouse y el teclado, se consideran en la actualidad totalmente obsoletos. Los usuarios se inclinan por ver y experimentar nuevas formas de interactuar como jugar, la presencia de elementos más visuales con una mayor interacción, y un mínimo de esfuerzo de interacción.

Producto de este estudio experimental de acople "sensor - producto multimedia" se recomienda que:

- Los desarrolladores de sistemas multimedia deben buscar un nuevo elemento innovador en el acople de control con el sistema de menú.

- Incorporar en una segunda etapa la grabación de más locaciones de la ciudad de Guayaquil.

- En el caso de uso de drones, se debe estar al día en las nuevas disposiciones y reglamentos para pilotear estos apararos de manera segura.

- Usar lenguajes de programación y entornos de desarrollo actuales para que el uso de los driver con los nuevos dispositivos tecnológicos funcionen de manera óptima.

- Hacer pruebas con la detección de voz para incorporarlo al control de un multimedio interactivo.

- Revisar todas las actividades pedagógicas que se desarrollan en la escuela y potenciar el uso de esta tecnología en la escuela y en casa.

- Estudio de mercado para la comercialización del software multimedia y su impacto como software de apoyo educativo.

- Actualización a los docentes de centros educativos en el uso de las nuevas tecnologías de la información y la comunicación. 
CONCLUSIONES

Al operacionalizar la tecnología sensores de movimiento, conectando los sensores a una computadora por su puerto USB, se elaboró un algoritmo - seudocódigo de instalación y operación para sistematizar el proceso de instalación y reconocimiento gestual a través de un flujograma, para que se pueda codificar en cualquier lenguaje de programación.

Se establecieron los parámetros técnicos requeridos para la producción de multimedios interactivos educativos, realizando una investigación bibliográfica y consulta a expertos, para formular una metodología de trabajo; por lo que los sistemas multimedia que usan los tradicionales dispositivos de control en sus sistemas de menú como el mouse y el teclado se consideran en la actualidad totalmente obsoletos, los usuarios se inclinan a ver y experimentar nuevas formas de interactuar como jugar, y elementos más visuales. Los parámetros son:

- Utilizar un componente de entrada de última tecnología interactivo de fácil manejo (Kinect).

- La comunicación usuario-software se realice a través de las partes del cuerpo (manos).

- Inclusión de contenidos y actividades didácticas e informativas que motiven el interés por las ciencias, las tecnologías y la investigación.

- Claridad del programa (grados de usabilidad)

Se produjeron 10 cortos educativos con guiones originales escritos por miembros del equipo investigador usando filmaciones normales y tomas áreas de drones. Se realizó el experimento de acople sensor-video y el acople de un producto multimedia, usando sensores de movimiento para controlar la galería de videos previamente producidos, para diseñar un software de Control Gestual. Se concluye que el uso de tomas aéreas para visualizar un mismo objeto o lugar que ha sido visto muchas veces produce nuevas experiencias en los usuarios, se evidencia que los mismos describen cosas nuevas no vistas cuando se lo visita físicamente, los productores deben tomar en consideración en las posproducción las tomas de plano general y panorámico como anticuadas. Se sugiere hacer un "mix" con nuevas tomas.

El impacto científico y social del uso de sensores de movimiento en los desarrollos de productos multimedia es alto, e implica que se tome en cuenta su aportación a la interactividad y la usabilidad del software, se sugiere experimentar con otras soluciones multimedia de corte social desarrollando multimedios de corte pedagógicos.

Como parte del experimento una de las dificultades se centra en las pruebas realizadas donde la cámara capta luz, lo que afecta en ciertos caso el reconocimiento de las manos, aunque no es una limitación importante debe ser considerada como parte de estudios futuros.

Para finalizar el estudio se deja planteada dos preguntas concernientes a los sensores de movimientos.

¿Cuál será el impacto de la realidad virtual y el uso de sensores de movimiento del tipo detección de manos como LeapMotion?.

¿Los productos multimedia seguirán la tendencia cambiante de la tecnología contemporánea de los métodos de hardware para su control innovando o evolucionando los sensores de movimientos a otro nivel?.

AGRADECIMIENTOS

Agradecer principalmente a Dios por haber permitido desarrollar el proyecto, a nuestras familias, al Instituto de Cultura, Arte, Idiomas y Multimedia de la Facultad de Artes y Humanidades, al Sistema de Investigación y Desarrollo de la Universidad Católica de Santiago de Guayaquil por su apoyo que siempre estuvieron asesorándonos para cumplir satisfactoriamente la culminación del proyecto.

MSc. Wellington Villota y MSc. Yamil Lambert

\section{REFERENCIAS BIBLIOGRÁFICAS}

Bou, G. (1997). El Guión Multimedia. Barcelona: Anaya Multimedia.

Cortes Rodríguez, J., Sandoval, F., y Trujillo Pascual, I. (2014). Implementación de un control gestual. México: Escuela Superior de Ingeniería Mecánica y Eléctrica.

Chuya Sumba, J., Cuenca Soto, M., y Delgado Guaraca, K. (2013). Diseño e implementación de un sistema para el análisis del movimiento humano usando sensores Kinect. Recuperado de : http://dspace. ups.edu.ec/handle/123456789/5092

Fernández, E. (2012). Control de Software Educativo Mediante Kinect de Microsoft. Madrid: Universidad Carlos III de Madrid. Obtenido de Carlos III de Madrid. Recuperado de: http://e-archivo.uc3m.es/ bitstream/handle/10016/16846/TFG_Estefania_Fernandez_Sanchez.pdf?sequence=1 
Figueroa Pedreros, J. R. (2012). Diseño de un Modelo de Interacción para la navegación en 3D, basado en la captura de gestos corporales por el Sensor Microsoft Kinect. Bogotá, Colombia: Universidad Militar Nueva Granada: Facultad de Ingeniería.

González de Dios, A. (2014). Desarrollo y evaluación de un sistema de teleoperación de robots basado en reconocimiento de gestos usando el sensor Kinect. Madrid: Universidad Politécnica de Madrid.

Gousset, M., Hinshelwood, M., Randell, B., Keller, B., \& Woodward, M. (2014). Application Licecycle Management with Visual Studio 2013. Indianapolis: Wrox.
Hejlsberg, A., Wiltamuth, S., \& Golde, P. (2006). The C\# Programming Language (2nd Edition ed.). Addison-Wesley Professional.

Jago, M. (2015). Adobe Premiere Pro CC Classroom in a Book ( 1st ed.). Adobe Press.

Rodríguez, J. (2013). Interacción humano-computador en escenarios educativos y artísticos. Kinect como propuesta viable. Revista Elementos, 3(3).

Sáenz, Valiente, (2008), Artey Técnica dela Animación, Buenos Aires.

Zubair Shah, P. (2009), Pakistan Says U.S. Drone Kills 13, New York Times. 